\title{
Persistent photoconductivity and optical quenching of photocurrent in GaN layers under dual excitation
}

\author{
V. V. Ursaki and I. M. Tiginyanu \\ Laboratory of Low-Dimensional Semiconductor Structures, Institute of Applied Physics, \\ Technical University of Moldova, Bd. Stefan cel Mare 168, Chisinau 2004, Moldova \\ P. C. Riccia) and A. Anedda \\ Dipartimento di Fisica, Università di Cagliari and INFM, UdR Cagliari, Cittadella Universitaria S.P. No. \\ 8, Km 0.700, 09042 Monserrato (Ca), Italy \\ S. Hubbard and D. Pavlidis \\ EECS Department, The University of Michigan, Ann Arbor, Michigan 48109-2122
}

(Received 28 January 2003; accepted 10 July 2003)

Persistent photoconductivity (PPC) and optical quenching (OQ) of photoconductivity (PC) were investigated in a variety of $n$-GaN layers characterized by different carrier concentrations, luminescence characteristics, and strains. The relation between PPC and OQ of PC was studied by exciting the samples with two beams of monochromatic radiation of various wavelengths and intensities. The PPC was found to be excited by the first beam with a threshold at $2.0 \mathrm{eV}$, while the second beam induces $\mathrm{OQ}$ of $\mathrm{PC}$ in a wide range of photon energies with a threshold at $1.0 \mathrm{eV}$. The obtained results are explained on the basis of a model combining two previously put forward schemes with electron traps playing the main role in PPC and hole traps inducing OQ of PC. The possible nature of the defects responsible for optical metastability of $\mathrm{GaN}$ is discussed. (C) 2003 American Institute of Physics. [DOI: 10.1063/1.1604950]

\section{INTRODUCTION}

Over the last years, spectacular progress was achieved in fabrication of $\mathrm{GaN}$ based short-wavelength light-emitting devices ${ }^{1-4}$. Besides that, GaN and related compounds offer great potential for applications in ultraviolet detectors ${ }^{5,6}$ and field effect transistors. ${ }^{7,8}$ The existence of metastable defects in $\mathrm{GaN}$ does not seem to have a negative impact on light emitters, while the PPC behavior associated with these defects can have a significant effect on the characteristics of FET and UV detectors based on AlGaN/GaN heterostructures, including sensitivity, noise properties, dark level, and response speed. ${ }^{7,9}$ Persistent photoconductivity (PPC) proves to be inherent to wurtzite-type $\mathrm{GaN}$ epitaxial layers. ${ }^{10}$ Different mechanisms were considered as the origin of PPC, such as defects with bistable character, ${ }^{10,11} A X,{ }^{12}$ or $D X$ (Refs. 13 and 14) centers, random potential fluctuations due to nonstoichiometry and heterointerfaces, ${ }^{15,16}$ and unintentionally incorporated cubic-phase crystallites in the hexagonal matrix. ${ }^{17}$ Other effects related to the PPC, such as yellow luminescence (YL), ${ }^{10,18,19}$ time dependent luminescence, ${ }^{20,21}$ quenching of photocurrent, ${ }^{21,22}$ and optical quenching (OQ) of photoconductivity $(\mathrm{PC})^{23,24}$ indicate that bistable defects are the most probable source of the optical metastability. Recently, the OQ phenomena were pointed out to be closely related to the defects responsible for the PPC effects ${ }^{24}$.

In spite of considerable efforts in investigating optical metastability in GaN, the explanation of the phenomenon is still contradictory. Particularly, the microscopic nature of the

${ }^{a)}$ Author to whom correspondence should be addressed; electronic mail: carlo.ricci@dsf.unica.it centers responsible for the effect involved remains uncertain, gallium vacancy $\left(V_{\mathrm{Ga}}\right),{ }^{11,24,25}$ nitrogen antisite $\left(\mathrm{N}_{\mathrm{Ga}}\right)$, $10,18,19,24$ and gallium antisite $\left(\mathrm{Ga}_{\mathrm{N}}\right)^{21}$ being the preferable candidates. The issue concerning the major role of electron ${ }^{18,19,22}$ or hole ${ }^{23-25}$ trapping processes in optical metastability of $\mathrm{GaN}$ is also under discussion.

The goal of this article is to investigate the relation between PPC and PC quenching phenomena in n-type GaN epilayers. For this purpose, PPC and OQ of PC were studied in a series of $\mathrm{GaN}$ epilayers with different carrier concentrations, luminescence characteristics, and strains. Sample characterization was carried out in the temperature interval from 10 to $300 \mathrm{~K}$ under simultaneous excitation by two monochromatic light beams of various wavelengths and intensities.

\section{EXPERIMENT}

The GaN layers investigated in this study were grown by low-pressure metalorganic chemical vapor deposition on (0001) $c$-plane sapphire or $6 \mathrm{H}-\mathrm{SiC}$ substrates using trimethylgallium and ammonia as source materials. ${ }^{26} \mathrm{~A}$ buffer layer of about $25 \mathrm{~nm}$ thick GaN was first grown at $510{ }^{\circ} \mathrm{C}$. Subsequently, $n$-GaN layers were grown at $1100{ }^{\circ} \mathrm{C}$. The thickness of the layers varies from several hundreds of nanometers to several tens of micrometers. According to Hall effect measurements, the room-temperature electron concentration in specially undoped layers is $\sim 10^{17} \mathrm{~cm}^{-3}$. Si-doped samples with electron concentration ranging from $10^{18}$ to $10^{19} \mathrm{~cm}^{-3}$ were also used in our experiments. For PC measurements, ohmic contacts were formed by depositing in- 


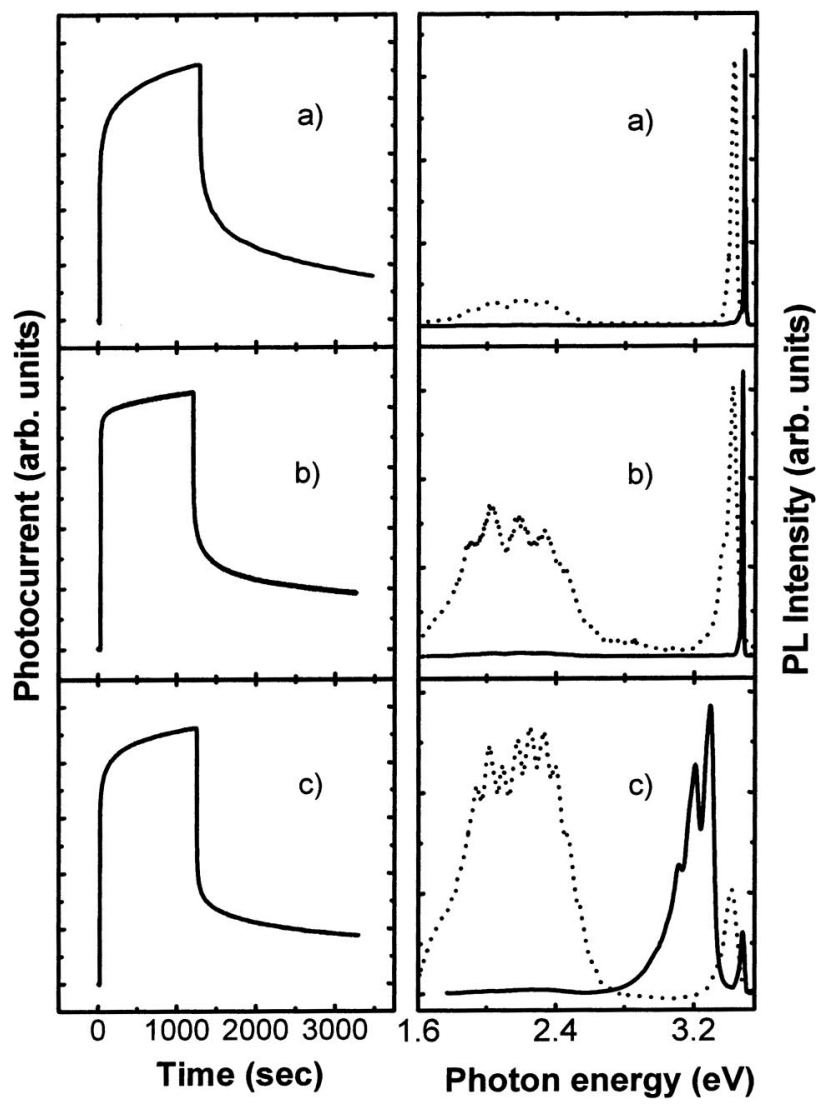

FIG. 1. Right-hand side: The PL spectra measured at $10 \mathrm{~K}$ (solid line) and $300 \mathrm{~K}$ (dashed line) for three representative $1.3 \mu \mathrm{m}$ thick $n$-GaN layers with different electron concentrations: Sample No. 2 (a), sample No. 4 (b), and sample No. 5 (c) in Table I. Left-hand side: The PC buildup and decay for the same layers measured at room temperature.

dium and annealing the samples at $400{ }^{\circ} \mathrm{C}$ for $10 \mathrm{~s}$. The radiation from xenon or mercury lamps passed through a monochromator, as well as different lines of an $\mathrm{Ar}^{+}$laser, were used for the excitation of PC. The samples were simultaneously irradiated by two beams of monochromatic radiation. One beam of radiation, called "source A", provides either intrinsic or extrinsic excitation with photon energy $h \nu_{\mathrm{A}}>2.0 \mathrm{eV}$. The second excitation source, called "source B", provides extrinsic excitation with photon energy $h \nu_{\mathrm{B}}$ $\left\langle h \nu_{\mathrm{A}}\right.$. The specimens were mounted on the cold station of LTS-22-C-330 workhorselike optical cryogenic system. The measurements were carried out in the temperature interval from 10 to $300 \mathrm{~K}$. Special care was taken to ensure the equilibrium value of the dark current in samples before each experiment.

\section{RESULTS}

The investigated $\mathrm{GaN}$ epitaxial layers show pronounced PPC independent of the carrier concentration, strain, or luminescence spectrum. Figure 1 illustrates this observation for three representative layers grown on sapphire substrates. The specially undoped layer [Fig. 1(a)] with the electron concentration $1 \times 10^{17} \mathrm{~cm}^{-3}$ exhibits only excitonic luminescence at $10 \mathrm{~K}$ temperature and an additional yellow PL band at room temperature. The moderately doped layer [Fig. 1(b)] with electron concentration $2 \times 10^{18} \mathrm{~cm}^{-3}$ shows (YL),

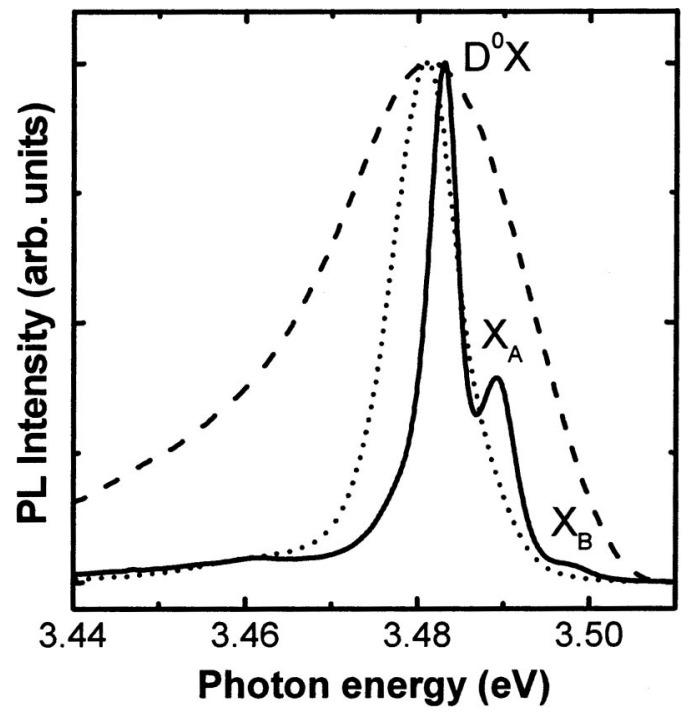

FIG. 2. Comparison of the excitonic PL spectra measured at $10 \mathrm{~K}$ for GaN layers with different electron concentrations: Sample No. 2 (solid line), sample No. 4 (dotted line), and sample No. 5 (dashed line) in Table I.

which is of low intensity at $10 \mathrm{~K}$ temperature, and it becomes comparable with the excitonic luminescence at room temperature. The luminescence spectrum of the highly doped layer with $n=10^{19} \mathrm{~cm}^{-3}$ is predominated by the UV photoluminescence (PL) band at $3.27 \mathrm{eV}$ exhibiting phonon replicas at low temperature and by the yellow band at room temperature. The PL band at $3.27 \mathrm{eV}$ is commonly observed in low-temperature PL spectra of GaN and is attributed to the donor-acceptor-pair transitions with $\mathrm{Si}$ impurity as a donor. ${ }^{27}$ Figure 2 presents the PL spectra of the same GaN layers in the excitonic region measured at $10 \mathrm{~K}$. One can see that at low electron concentration, the PL peaks associated with the recombination of $D^{0} X, X_{A}$, and $X_{B}$ excitons are well resolved. With increasing the electron concentration, the excitonic PL bands broaden and overlap. This broadening is attributed to tailing of the density of states caused by potential fluctuations due to randomly distributed impurities. It can be quantitatively explained using the impurity band broadening model proposed by Morgan. ${ }^{28,29}$

The energy position of the excitonic PL band is nearly the same in the three samples illustrated in Fig. 1 and it is shifted by $\sim 10 \mathrm{meV}$ toward high energies in comparison with the position of respective excitons in non-stressed GaN layers. ${ }^{30}$ It means that considerable compressive strains exist in our $\mathrm{GaN}$ layers due to the mismatch between epilayers and sapphire substrates. Using the previously reported rates of the exciton line shifts with the biaxial stress in $\mathrm{GaN},{ }^{30}$ the strains in our $1.3 \mu \mathrm{m}$ thick layers were estimated to be as high as $0.5 \mathrm{GPa}$. Note that we have also investigated $\mathrm{GaN}$ layers with the thickness ranging from 0.5 to $20 \mu \mathrm{m}$ grown on sapphire substrates, as well as layers grown on $\mathrm{SiC}$ substrates. In the last case, the exciton PL band proves to be shifted toward low energies indicating that the layers suffer from tensile strain. Note that we observed no clear correlation between PPC and any parameters of the investigated layers such as electron concentration, strain, or YL intensity (see Table I). 
TABLE I. Parameters of GaN layers studied in this work.

\begin{tabular}{lccccccc}
\hline \hline $\begin{array}{l}\text { Sample No. } \\
\text { substrate }\end{array}$ & $\begin{array}{c}\text { Thickness } \\
(\mu \mathrm{m})\end{array}$ & $n\left(10^{18} \mathrm{~cm}^{-3}\right)$ & $\begin{array}{c}\text { Shift of the } \\
\text { exciton line } \\
(\mathrm{meV})\end{array}$ & $\begin{array}{c}\text { Estimated } \\
\text { strain }(\mathrm{GPa})\end{array}$ & YL at $300 \mathrm{~K}$ & $I_{\mathrm{PC}} / I_{\text {dark }}$ & $\begin{array}{c}\text { PCC time } \\
\text { constant }(\mathrm{s})\end{array}$ \\
\hline 1 Sapphire & 0.5 & 0.1 & +20 & 1 compres. & weak & 0.8 & 420 \\
2 Sapphire & 1.3 & 0.1 & +10 & 0.5 compres. & very weak & 0.6 & 300 \\
3 Sapphire & 1.3 & 0.6 & +10 & 0.5 compres. & weak & 0.2 & 110 \\
4 Sapphire & 1.3 & 2.0 & +10 & 0.5 compres. & moderate & 1.6 & 100 \\
5 Sapphire & 1.3 & 10.0 & +10 & 0.5 compres. & strong & 0.9 & 90 \\
6 Sapphire & 4.5 & 0.1 & +6 & 0.3 compres. & very weak & 0.7 & 320 \\
7 Sapphire & 20.0 & 0.1 & +2 & 0.1 compres. & very weak & 0.4 & 210 \\
8 SiC & 0.5 & 1.0 & -16 & 0.8 tensile & strong & 0.6 & 420 \\
9 SiC & 1.2 & 1.0 & -8 & 0.4 tensile & moderate & 1.2 & 510 \\
10 SiC & 1.2 & 4.0 & -8 & 0.4 tensile & strong & 0.7 & 310 \\
11 SiC & 4.0 & 1.0 & -4 & 0.2 tensile & moderate & 0.3 & 80 \\
\hline \hline
\end{tabular}

The data presented in Fig. 1 indicate no correlation between YL and PPC in our samples. This issue is widely discussed in literature. No relationship has been evidenced by Beady et al. ${ }^{15}$ Hirsch et al.,${ }^{16}$ and Ptak et al. ${ }^{31}$ According to other authors, ${ }^{10,18,19}$ PPC and YL phenomena in GaN are related to each other via an intrinsic defect. Recently, Boglio et al. ${ }^{32}$ found a correlation between transient dynamics of the PC and yellow PL defects in GaN samples but no correlation was evidenced between the strength of yellow PL and the magnitude of the PPC effect. We consider that a possible explanation of the scatter in the experimental data is the nonelementary character of both YL and PPC. It was suggested recently that several electron transitions contribute to the yellow PL. ${ }^{31}$ One of these transitions is related to the defect responsible for PPC, while the other ones are related to additional impurities or defects. The analysis of the photocurrent transients in Fig. 1 also indicates the complicated nature of the PPC effect. Our samples prove to exhibit several PPC components. Usually, the PPC decay is described either by the Gaussian distribution of capture barriers, ${ }^{16}$ or using the so-called "stretched exponential": $:^{13,19,25}$

$$
G(t)=G(0) \exp \left[\left(t / \tau_{\mathrm{PPC}}\right)^{\beta}\right],
$$

where $G(0)$ is the PPC buildup level at the moment of light excitation being removed, $\tau_{\mathrm{PPC}}$ is the PPC decay time constant, and $\beta$ is the decay exponent.

At least two components are necessary for a satisfactory stretched exponential fit of the PPC decay in our samples. Figure 3 illustrates this observation for the PPC decay measured at room temperature in a specially nondoped $\mathrm{GaN}$ layer. The experimental data are better fitted with the value of $\beta$ equaling 0.26 at the decay time up to $1000 \mathrm{~s}$. This value of $\beta$ is very close to that reported by Li et al. ${ }^{12}$ and Chen $e t$ al.. ${ }^{18,19}$ At decay times higher than $1000 \mathrm{~s}$, better fit is achieved with $\beta=0.37$ that corresponds to the value found by Qiu and Pankove. ${ }^{25}$ Note that two-time regimes of PPC in GaN were recently attributed ${ }^{31}$ to different charge states of the same native defect which can trap carriers with different release times.

The PC decay and the transient dynamics of the PC in $\mathrm{GaN}$ and related compounds is well studied. ${ }^{13,14,16,18,19,24,25,31,32}$ However, as mentioned in Sec. I, there is no general consensus concerning the mechanism re- sponsible for the PPC in these materials. Apart from that, the mechanisms of PPC in $\mathrm{AlGaN} / \mathrm{GaN}$ heterostructures and bulk $\mathrm{GaN}$ could be different. PPC in $\mathrm{AlGaN} / \mathrm{GaN}$ heterostructures is believed to come from the $D X$ centers in the AlGaN layer, ${ }^{9,13,14}$ while in bulk $\mathrm{GaN}$, the preference is given to vacancies ${ }^{21,24,25,31,32}$ and/or antisite-type defects. ${ }^{10,18,19,21,24}$ One model was invoked to explain the separation of carriers from traps by random local-potential fluctuations that reduce the recapture rate of the traps and give rise to PPC. ${ }^{16,33}$ The main sources of local-potential fluctuations are the nonuniform impurity distribution in highly doped and compensated semiconductors, and the strains in films grown on substrates with high lattice mismatch. Since the PPC is observed in layers with different dopant concentrations and strains, we consider that the aforementioned mechanism is less probable in comparison with the mechanism involving bistable defects. In order to investigate the distribution of the metastable defect energy levels in the band gap of GaN layers, we have carried out experiments by exciting samples with two beams of radiation.

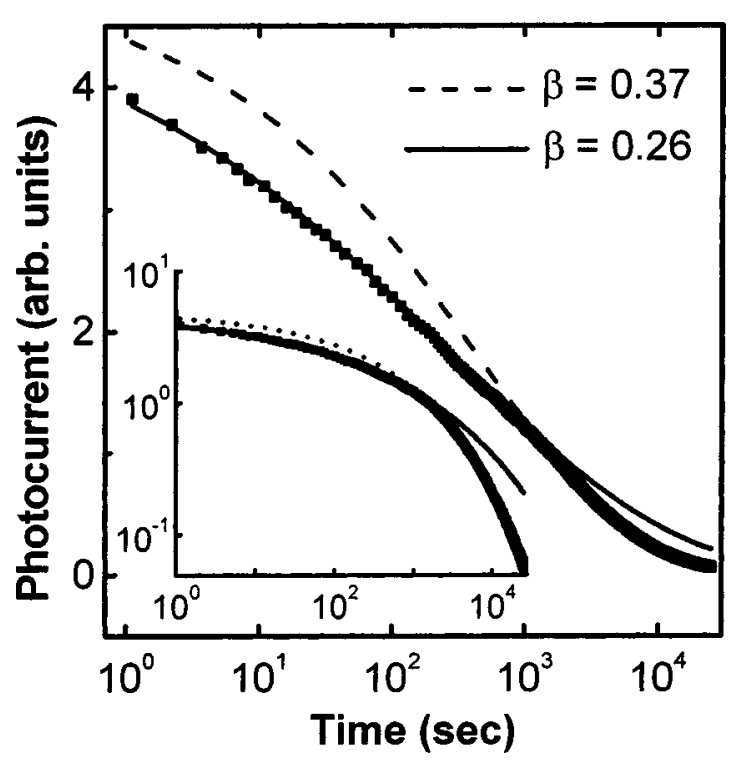

FIG. 3. Experimental PPC decay at $300 \mathrm{~K}$ and stretched exponential fit for a specially nondoped GaN layer (sample No. 2 in Table I). Inserted is the same in a double logarithmic scale. 


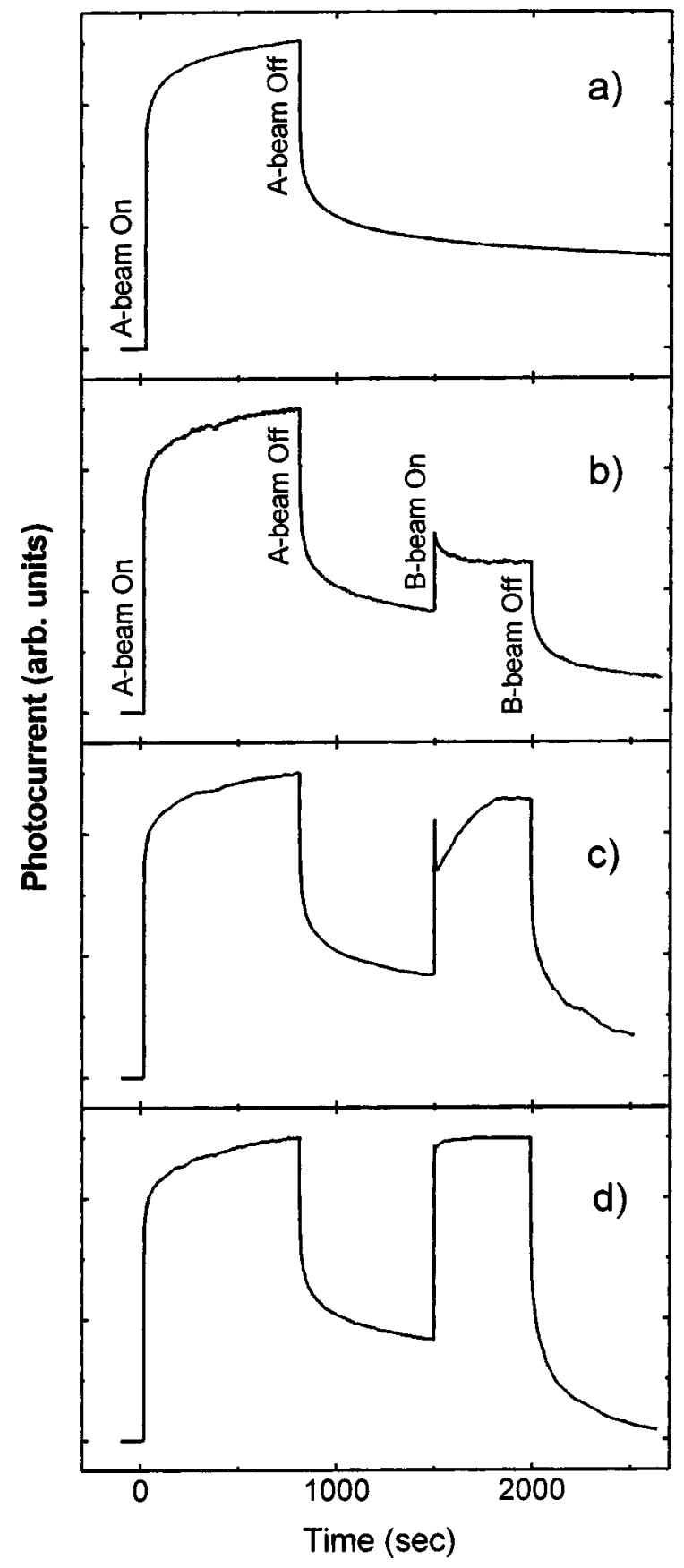

FIG. 4. PPC kinetics under excitation with $365 \mathrm{~nm}$ line followed by the illumination with the $546 \mathrm{~nm}$ line at $300 \mathrm{~K}$ (sample No. 4 in Table I). The intensity of the $365 \mathrm{~nm}$ beam is the same in (a)-(d), while the intensity of the $546 \mathrm{~nm}$ beam is zero in (a) and increases from (b) to (d).

Figure 4 illustrates the PPC dynamics in the GaN layer No. 4. Note that the trends discussed next for this sample are inherent to all specimens from Table I. After illumination with intrinsic excitation by the 365 line of xenon lamp (excitation A), pronounced PPC is observed [Fig. 4(a)]. In Figs. 4(b) $-4(d)$, the intrinsic excitation is followed by the extrinsic one with the $546 \mathrm{~nm}$ line (excitation B). The intensity of the $365 \mathrm{~nm}$ excitation is the same in all cases, while the intensity of the $546 \mathrm{~nm}$ excitation increases from Figs. 4(b) to 4(d). As can be seen, the extrinsic B-excitation leads again to PC. However, when the B source is turned off, the PC decays to

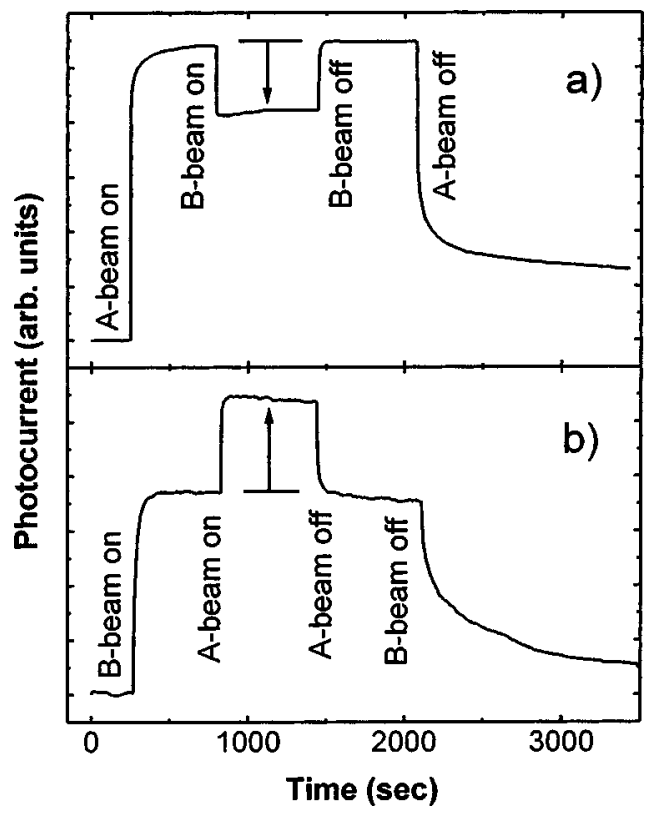

FIG. 5. The PC kinetics in a GaN layer (sample No. 4 in Table I) subjected for a period of time to simultaneous excitation with two beams of monochromatic radiation at $300 \mathrm{~K}$. The A beam corresponds to $365 \mathrm{~nm}$ line of a mercury lamp, while the $\mathrm{B}$ beam is the $514 \mathrm{~nm}$ line of an $\mathrm{Ar}^{+}$laser. The arrows indicate the evolution of the PC by increasing the intensities of the $\mathrm{B}$ beam (a) and A beam (b).

a value appreciably lower than that observed when applying only the intrinsic A excitation, see Fig. 4(a).

In the case of simultaneous excitation with sources $\mathrm{A}$ and $\mathrm{B}$, the $\mathrm{B}$ excitation leads to partial quenching of $\mathrm{PC}$ induced by the A excitation [see Fig. 5(a)]. On the other hand, the A excitation always contributes to PC but never to quenching [see Fig. 5(b)]. The character of the contribution of the sources A and B does not depend upon the sequence of illuminating the sample with light beams. In Fig. 5(a), the A beam is applied to the sample first, and the subsequent excitation with the $\mathrm{B}$ beam induces OQ. In Fig. 6a, on the contrary, the excitation by the B beam is applied first, it being followed by the switch on of the A beam. In such a case, the OQ is evidenced once again when switching off the B beam. The sequence of illuminating the sample with $A$ and B light beams is opposite in Figs. 5(b) and 6(b), nevertheless, the A beam leads to PC in both cases.

Figure 7 presents the spectral distribution of the PPC and OQ of PC measured in sample No. 2 at room temperature. The PC was excited by Xe lamp light passed through a monochromator. In the $\mathrm{OQ}$ experiments, the $\mathrm{PC}$ is excited by the $365 \mathrm{~nm}$ line of a $\mathrm{Hg}$ lamp as primary source, and the OQ is induced by the Xe-lamp light passed through a monochromator as secondary source. The PPC is observed for photon energies $h \nu_{\mathrm{A}}>2.0 \mathrm{eV}$ [Fig. 7(a)]. The OQ occurs for photon energies $1.0 \mathrm{eV}<h \nu_{\mathrm{B}}<2.6 \mathrm{eV}$ [Fig. 7(b)]. As just mentioned, the OQ is inherent to all specimens from Table I. It occurs in the temperature interval from 10 to $300 \mathrm{~K}$ providing that the photon energies of the light sources satisfy the relations: (i) $h \nu_{\mathrm{A}}>2.0 \mathrm{eV}$; (ii) $1.0 \mathrm{eV}<h \nu_{\mathrm{B}}<2.6 \mathrm{eV}$; (iii) $h \nu_{\mathrm{B}}<h \nu_{\mathrm{A}}$. The photon energy interval of $\mathrm{B}$ excitation can be divided into two parts. The B excitation always leads to OQ for 


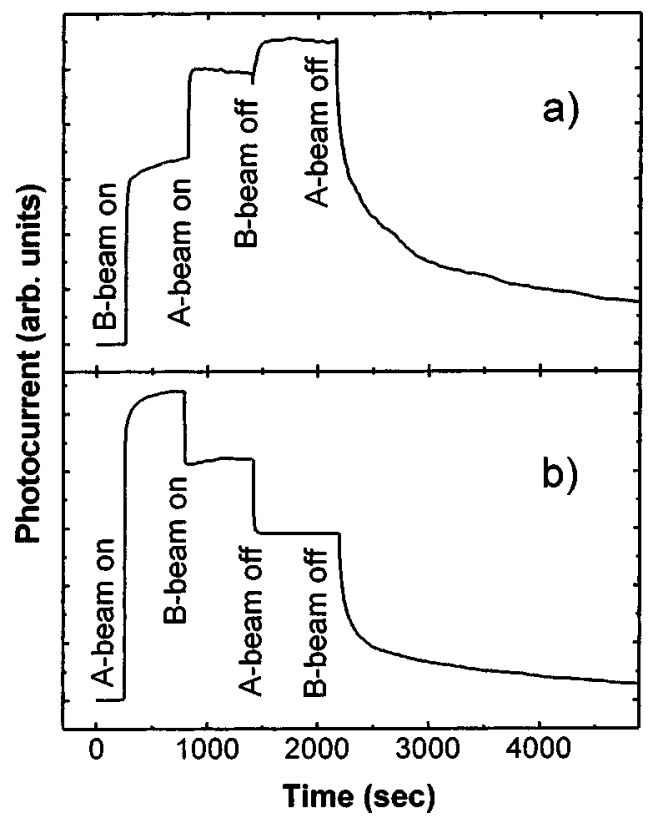

FIG. 6. The same as Fig. 5, but with inverse order of applying the excitation beams.

photon energies $1.0 \mathrm{eV}<h \nu_{\mathrm{B}}<2.0 \mathrm{eV}$, while for $2.0 \mathrm{eV}$ $<h \nu_{\mathrm{B}}<2.6 \mathrm{eV}$, an additional condition should be satisfied for OQ to occur, namely the intensity of the B beam should be lower than a critical $\left(I_{c}\right)$ value. When this condition is not satisfied, the B excitation induces PPC rather than OQ (see Fig. 8).

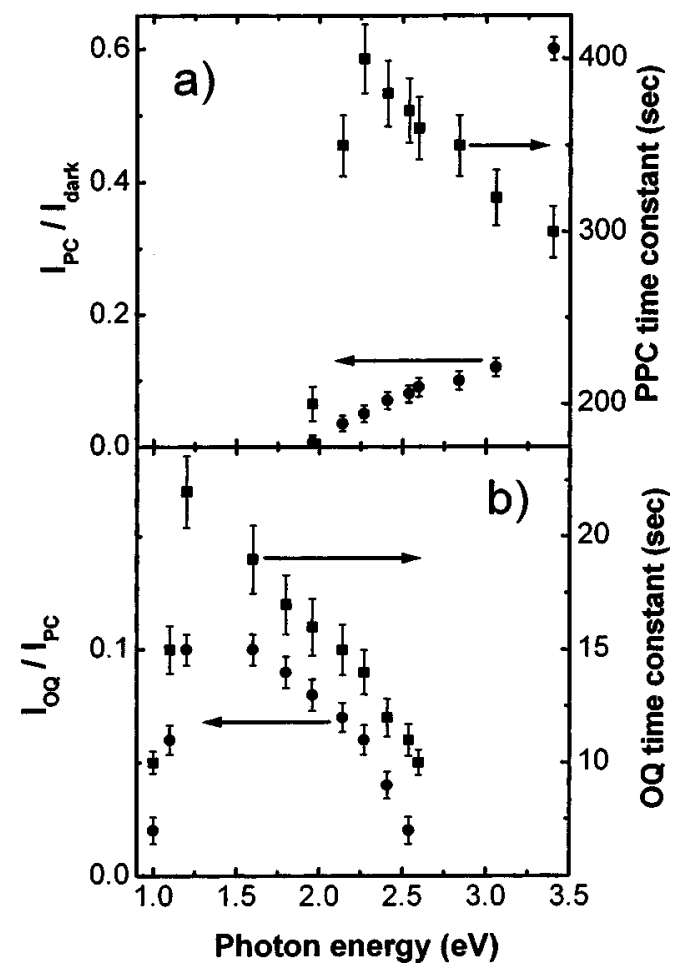

FIG. 7. Spectral dependence of the PPC (a) and OQ (b) measured in sample No. 2 at $300 \mathrm{~K}$. The spectral resolution is $\sim 20 \mathrm{meV}$.

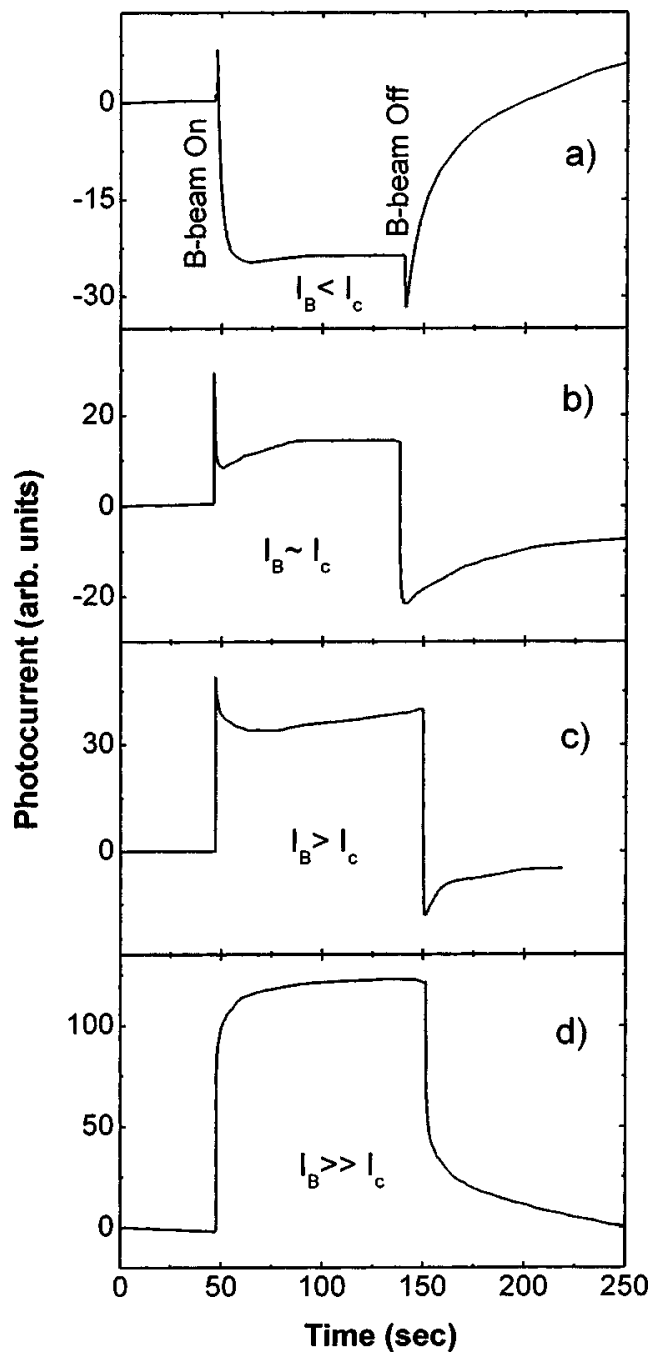

FIG. 8. Effect of the excitation density of secondary source (514 nm line of an $\mathrm{Ar}^{+}$laser, source B) on the PC generated by a primary source $(365 \mathrm{~nm}$ line of a mercury lamp, source A) in sample No. 4 at $300 \mathrm{~K}$. The sample is illuminated by the primary source during the period of time from 0 to $250 \mathrm{~s}$. The zero level of photocurrent axis corresponds to the stationary PC induced by the A beam.

\section{DISCUSSION}

Several studies have been performed to explain the origin of OQ, PPC, and YL in GaN. According to some models, ${ }^{10,18,19}$ PPC and YL may be attributed to the same defects. Lin $e t a l .{ }^{24}$ proposed that the origin of OQ is closely related to those defects, too. Nevertheless, the situation remains uncertain and rather confusing. Particularly, different schemes concerning the major role of electron ${ }^{18,19,22}$ or hole ${ }^{23-25,31,32}$ traps in these processes are currently under consideration. The microscopic nature of the defects responsible for the phenomena involved is also under discussion. The reported values for the threshold photon energy to observe PPC vary in a rather wide spectral range from $1.1 \mathrm{eV}$ (Refs. 25 and 31) to $2.3 \mathrm{eV}$ (Refs. 18 and 19). The analysis of the spectral distribution of the OQ suggests that the phenomenon is related to defects with energy levels distributed from 0.9 to $2.6 \mathrm{eV}$ above the valence band. ${ }^{24}$ The broad spectral range of photon illumination leading to PPC and OQ phenomena is strong evidence that not a single defect is re- 

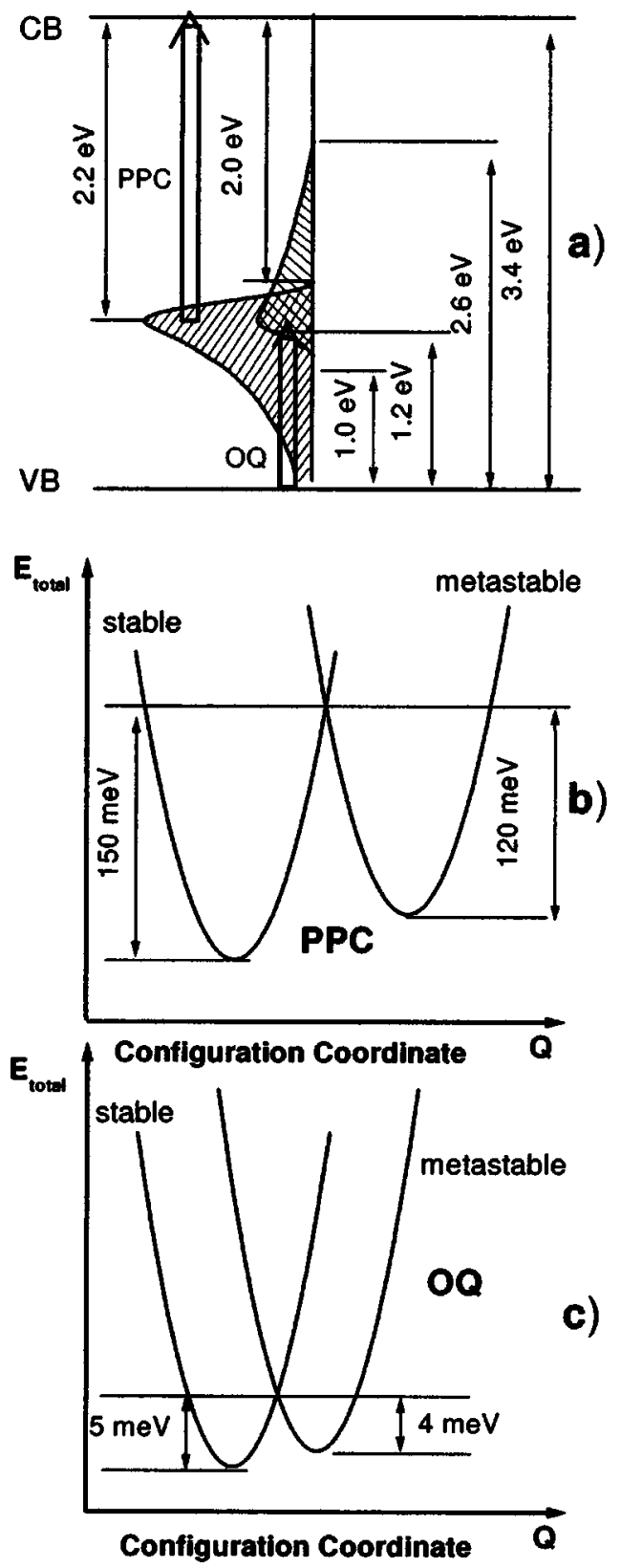

FIG. 9. Scheme of the density of state distribution and electron transitions accounting for PPC and OQ effects in GaN layers (a). Configurationcoordinate diagram of the defects responsible for PPC (b) and OQ (c) in GaN layers.

sponsible for the optical metastability of $\mathrm{GaN}$. On the basis of the obtained experimental data, we suppose that at least the defects responsible for PPC are different from those related to OQ. The PPC is excited according to the scheme proposed in Refs. 10,18,19 and 22, in which the electron trapping processes play the main role. The excitation of PPC over a broad range of photon energies starting from $2.0 \mathrm{eV}$ in our experiments indicates that PPC is related to a defect level which is broad in nature, and is located approximately at $E_{c}-2.0 \mathrm{eV}$ with a distribution of states due to its complex structure. The maximum in the distribution of density of states is located near $E_{c}-2.2 \mathrm{eV}$ [see Fig. 9(a)]. The threshold of PPC excitation in our experiments is higher than that reported by Reddy et al. ${ }^{10}$ and Chung et al. ${ }^{22}$ but is lower than that observed by Chen et al. ${ }^{18,19}$ After an electron is excited from the defect level to the conduction band, the deep center can undergo a large lattice relaxation, which creates an energy barrier to prevent the photoexcited carrier from returning to the original state, thus, the PPC effect occurs [see the configuration-coordinate diagram in Fig. 9(b)]. The OQ effects appear to occur in accordance with the scheme proposed by Qiu and Pankove ${ }^{25}$ and Lin et al. ${ }^{24}$ with the hole traps playing the major role. The spectral distribution of the OQ effect with the threshold at $1.0 \mathrm{eV}$ is indicative of the existence of a defect level also broad in nature and located approximately at $E_{v}+1.0 \mathrm{eV}$ with a maximum in the distribution of density of states at $E_{v}+1.2 \mathrm{eV}$ [see Fig. 9(a)]. When the GaN sample is illuminated by photons with relevant energies, the electrons from the valence band are excited into the hole trap. Subsequently, the defects that trap electrons relax into a configuration which generates an energy barrier [see the configuration-coordinate diagram in Fig. 9(b)], and the recombination of the trapped electrons with the released holes is prohibited, thus, the released free holes can recombine with electrons from the conduction band which leads to the quenching of PC.

The situation is relatively simple when $h \nu_{\mathrm{A}}>2.0 \mathrm{eV}$ and $1.0 \mathrm{eV}<h \nu_{\mathrm{B}}<2.0 \mathrm{eV}$. In such a case, the A excitation excites the PPC, while the $\mathrm{B}$ excitation quenches it, i.e., induces OQ. When $h \nu_{\mathrm{B}}>2.6 \mathrm{eV}$, both light sources contribute to the same process of exciting PPC, and no OQ occurs. The occurring processes are more complicated when the B-source quanta energy lies in the interval $2.0 \mathrm{eV}<h \nu_{\mathrm{B}}<2.6 \mathrm{eV}$. In such a case, the A source continues to excite PPC, while the B source can contribute to the excitation of both the PPC and OQ. Several factors then determine the process prevailing under B excitation: (i) the intensity of the excitation beams; (ii) the distribution of the density of states of the two types of defects; and (iii) the rates of the PPC and OQ processes. The behavior of our samples under the $\mathrm{B}$ excitation indicates that the density of states of the defects responsible for the OQ is lower than that inherent to defects inducing PPC [see Fig. 9(a)], while the rate of the OQ process is higher than the rate of the PPC process. In such a case, the OQ predominates when the B-source intensity is lower than a critical $\left(I_{c}\right)$ value. With increasing the $\mathrm{B}$-excitation power density $\left(I_{\mathrm{B}}\right.$ $>I_{c}$ ), a saturation effect occurs in the filling of the states responsible for OQ phenomena which leads to the predomination of the PPC over the OQ. In the intermediate case when $I_{\mathrm{B}} \sim I_{c}$, the excitation channels compete with each other resulting in transient instabilities as illustrated in Fig. 8.

Note the interesting behavior of the sample when the A excitation is followed by the B one (see Fig. 4). After the A excitation, a part of the defects responsible for the PPC are in the metastable state undergoing a large lattice relaxation. Under the action of the subsequent $\mathrm{B}$ excitation, these defects are reconfigured to the original state and the PC decays to a value appreciably lower than that induced by only A excitation. Since the B excitation in Fig. 4 satisfies the condition $2.0 \mathrm{eV}<h \nu_{\mathrm{B}}<2.6 \mathrm{eV}$, the sample exhibits some instabilities as just discussed.

Let us discuss now the possible nature of defects giving rise to the PPC and OQ phenomena presented in this work. 


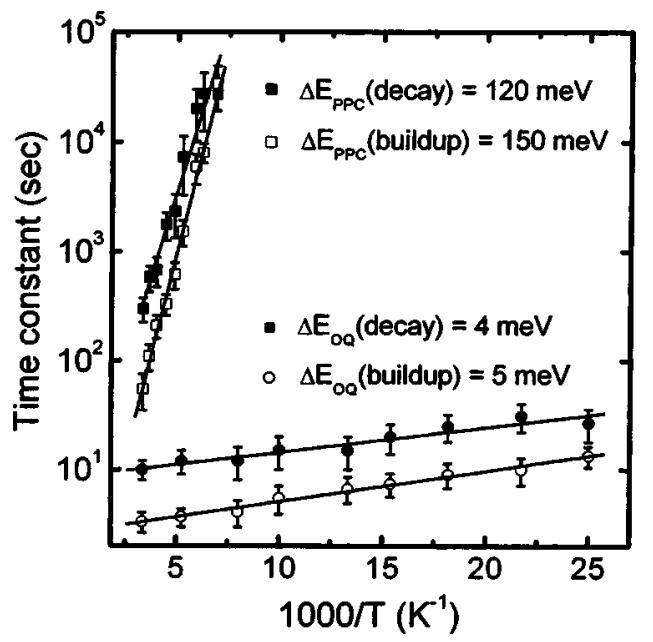

FIG. 10. The Arrhenius plot of the time constants for the PPC and OQ effects. The straight lines are the linear least-squares fit of the experimental data.

Our results indicate that the defects responsible for the PPC and OQ effects are broad in nature in agreement with previous observations. ${ }^{10,24}$ The steady increase of the photocurrent over a broad range of photon energies is in contrast with the photocurrent rise expected for the $D X$ or AX centers. If PPC is related to a $D X$-centerlike defect, a steep rise in the photocurrent should occur when the photon energy equals the ionization energy of the defect. Hence, the $D X$ centers as the origin of PPC and OQ effects in our samples are less probable. According to the previous reports, ${ }^{10,18,19,24,25,31,32}$ Gallium vacancy $\left(\mathrm{V}_{\mathrm{Ga}}\right)$ or nitrogen antisite $\left(\mathrm{N}_{\mathrm{Ga}}\right)$ is proposed as possible a defect which gives rise to PPC and OQ effects in $\mathrm{GaN}$ layers. Usually, both effects are considered to arise from the same defect. The aforementioned experimental data more likely indicate that one of these defects is responsible for PPC, while another one leads to OQ. The carrier capture barriers $\Delta E$ for the PPC and OQ obtained from the temperature dependence of $\tau$,

$$
\tau=\tau_{0} \exp [\Delta E / k T]
$$

are quite different for the two processes involved (see Fig. 10). The energy barrier between the metastable and original states of the defect responsible for PPC is by a factor of 30 higher than the respective one for the OQ (see Fig. 9). On the basis of this observation and taking into account that an antisite is more likely to undergo structural relaxation ${ }^{34,35}$ than a mere vacancy, it seems reasonable to assume that a nitrogen antisite (as proposed by Chen et al. $)^{18,19}$ or a defect complex like $\mathrm{N}_{\mathrm{Ga}}-\mathrm{Ga}_{i}$ (as proposed by Reddy et al. $)^{10}$ gives rise to PPC, while $\mathrm{V}_{\mathrm{Ga}}$ (Refs. 25 and 36) is responsible for OQ (as suggested by Lin et al.). ${ }^{24}$ This assumption is coherent with recent theoretical calculations. ${ }^{34-36}$ However, additional investigations with complementary methods, such as electron paramagnetic resonance, optically detected magnetic resonance, etc., are necessary for a reliable determination of the microscopic origin of the localized states responsible for PPC, OQ, and YL phenomena in GaN layers.

\section{CONCLUSION}

Since no correlations were found between the magnitude of the PPC effect in GaN layers and the potential fluctuations generated by the nonuniform impurity distribution and strains at heterointerfaces, the bistable intrinsic defects are believed to be the origin of optical metastability in gallium nitride.

The relation between PPC and OQ of PC evidenced in experiments with two simultaneously applied sources of monochromatic excitation are best described by a model combining two previously suggested schemes of PPC and OQ processes. The PPC effect is associated with electron traps located at $E_{c}-2.0 \mathrm{eV}$, while $\mathrm{OQ}$ of PC arises from hole traps with the energy level near $E_{v}+1.0 \mathrm{eV}$. The energy range of quanta exciting both PPC and OQ of PC reflects the broad distribution of the density of states due to the complex nature of the defects giving rise to optical metastability in $\mathrm{GaN}$. The nonelementary character of the PPC and OQ decay are indicative of multiple charge states of defects responsible for PPC and OQ effects. The analysis of the energy barriers between the stable and metastable states shows that the defect associated with PPC effect undergoes a much larger structural transformation than the one related to OQ phenomena. Taking into account the results of previous theoretical studies, ${ }^{34-36}$ we suggest that the deep defect involved in PPC is related to the nitrogen antisite, while the defect responsible for OQ can be attributed to the gallium vacancy.

\section{ACKNOWLEDGMENTS}

This work was supported by the U. S. Civilian Research and Development Foundation and Moldavian Research and Development Foundation under Grant No. ME2-3013.

${ }^{1}$ I. Akasaki, H. Amano, M. Kito, and K. Hiramatsu, J. Lumin. 48, 666 (1991).

${ }^{2}$ S. Nakamura, T. Mukai, and M. Senoh, Appl. Phys. Lett. 64, 1687 (1994).

${ }^{3}$ H. Morkoc, S. Strite, G. B. Gao, M. E. Lin, B. Sverdlov, and M. Burns, J. Appl. Phys. 76, 1363 (1994).

${ }^{4}$ S. Nakamura, S. Senoh, N. Iwasa, S. Nagahama, T. Yamada, T. Mutsushita, H. Kiyoku, and Y. Sugimoto, Jpn. J. Appl. Phys., Part 2 35, L74 (1996).

${ }^{5}$ G. Y. Xu, A. Salvador, W. Kim, Z. Fan, C. Lu, H. Tang, H. Morkoc, G. Smith, M. Estes, B. Goldenberg, W. Yang, and S. Krishnankutty, Appl. Phys. Lett. 71, 2154 (1997).

${ }^{6}$ A. Osinsky, S. Gangopadhyay, R. Gaska, B. Williams, M. A. Khan, D. Kuksenkov, and H. Temkin, Appl. Phys. Lett. 71, 2334 (1997).

${ }^{7}$ S. N. Mohammad, Z.-F. Fan, A. Salvador, O. Aktas, A. E. Botchkarev, W. Kim, and H. Morkoc, Appl. Phys. Lett. 69, 1420 (1996).

${ }^{8}$ M. A. Khan, Q. Chen, C. J. Sun, J. W. Yang, M. S. Shur, and H. Park, Appl. Phys. Lett. 68, 514 (1996).

${ }^{9}$ J. Z. Li, J. Y. Lin, H. X. Jiang, and M. A. Khan, Appl. Phys. Lett. 72, 2868 (1998).

${ }^{10}$ C. V. Reddy, A. Balakrishnan, H. Okumura, and S. Yoshida, Appl. Phys. Lett. 73, 244 (1998).

${ }^{11}$ C. Johnson, J. Y. Lin, H. X. Jiang, M. A. Khan, and C. J. Sun, Appl. Phys. Lett. 68, 1808 (1996).

${ }^{12}$ J. Z. Li, J. Y. Lin, H. X. Jiang, A Salvador, A. Botchkarev, and H. Morkoc, Appl. Phys. Lett. 69, 1474 (1996).

${ }^{13}$ J. Z. Li, J. Y. Lin, H. X. Jiang, M. A. Khan, and Q. Chen, J. Appl. Phys. 82, 1227 (1997).

${ }^{14}$ J. Z. Li, J. Y. Lin, H. X. Jiang, M. A. Khan, and Q. Chen, J. Vac. Sci. Technol. B 15, 1117 (1997). 
${ }^{15}$ G. Beadie, W. S. Rabinovich, A. E. Wickenden, D. D. Koleske, S. C. Binari, and J. A. Freitas, Jr., Appl. Phys. Lett. 71, 1092 (1997).

${ }^{16}$ M. T. Hirsch, J. A. Wolk, W. Walukiewicz, and E. E. Haller, Appl. Phys. Lett. 71, 1098 (1997).

${ }^{17}$ W. Rieger, R. Dimitrov, D. Brunner, E. Rohrer, O. Ambacher, and H. Stutzmann, Phys. Rev. B 54, 17596 (1996).

${ }^{18}$ H. M. Chen, Y. F. Chen, M. C. Lee, and M. S. Feng, Phys. Rev. B 56, 6942 (1997).

${ }^{19}$ H. M. Chen, Y. F. Chen, M. C. Lee, and M. S. Feng, J. Appl. Phys. 82, 899 (1997).

${ }^{20}$ I. K. Shmagin, J. F. Muth, J. H. Lee, R. H. Kolbas, C. M. Balkas, Z. Sitar, and R. F. Davis, Appl. Phys. Lett. 71, 455 (1997).

${ }^{21}$ S. Dhar and S. Ghosh, Appl. Phys. Lett. 80, 4519 (2002).

${ }^{22}$ S. J. Chung, M. S. Jeong, O. H. Cha, C.-H. Hong, E.-K. Suh, H. J. Lee, Y.

S. Kim, and B. H. Kim, Appl. Phys. Lett. 76, 1021 (2000).

${ }^{23}$ Z. C. Huang, D. B. Mott, P. K. Shu, R. Zhang, J. C. Chen, and D. K. Wickenden, J. Appl. Phys. 82, 2707 (1997).

${ }^{24}$ T. Y. Lin, H. C. Yang, and Y. F. Chen, J. Appl. Phys. 87, 3404 (2000).

${ }^{25}$ C. H. Qiu and J. I. Pankove, Appl. Phys. Lett. 70, 1983 (1997).
${ }^{26}$ S. M. Hubbarad, D. Pavlidis, V. Valiaev, M. A. Stevens-Kalceff, and I. M. Tiginyanu, Mater. Sci. Eng., B 91, 336 (2002).

${ }^{27}$ P. W. Yu, C. S. Park, and S. T. Kim, J. Appl. Phys. 89, 1692 (2001).

${ }^{28}$ T. N. Morgan, Phys. Rev. 139, A343 (1965).

${ }^{29}$ E. Illiopoulos, D. Doppalapudi, H. M. Ng, and T. D. Moustakas, Appl. Phys. Lett. 73, 375 (1998).

${ }^{30}$ See, for instance, B. Gil, in Gallium Nitride (GaN) II, Semiconductors and Semimetals 57, edited by J. P Pankove and T. D. Moustakas (Academic, New York, 1999), p. 209 and references therein.

${ }^{31}$ A. J. Ptak, V. A. Stoica, L. J. Holberg, M. Moldovan, and T. H. Myers, MRS Internet J. Nitride Semicond. Res. 5S1, w 11.45 (2000).

${ }^{32}$ A. Boglio, G. Traetta, M. Lomascolo, A. Passaseo, and R. Cingolani, J. Appl. Phys. 89, 5782 (2001).

${ }^{33}$ M. K. Sheinkman and A. Y. Shik, Sov. Phys. Solid State 10, 128 (1976).

${ }^{34}$ T. Mattila, A. P. Seitsonen, and R. M. Nieminen, Phys. Rev. B 54, 1474 (1996).

${ }^{35}$ I. Gorczyca, A. Svane, and N. E. Christensen, Phys. Rev. B 60, 8147 (1999).

${ }^{36}$ J. Neugebauer and C. G. Van de Walle, Appl. Phys. Lett. 69, 503 (1996). 